\title{
DGZI Implant Dentistry Award 2016
}

Die Deutsche Gesellschaft für Zahnärztliche Implantologie (DGZI) verleiht den im Jahre 2005 durch den Wissenschaftlichen Beirat der DGZI inaugurierten „DGZI Implant Dentistry Award“" anlässlich des 46. Internationalen DGZI Jahreskongresses in München vom 30. September bis 1. Oktober 2016 .

Der DGZI Implant Dentistry Award wird zur Würdigung einer wegweisenden wissenschaftlichen Arbeit auf dem Gebiet der Implantologie verliehen. Er stellt die höchste Auszeichnung einer wissenschaftlichen Leistung durch die DGZI dar und ist zurzeit mit insgesamt $10000 €$ dotiert. Der Preis wird national und international ausgeschrieben. Es können sich alle in Deutschland tätigen Zahnärzte, Oralchirurgen, Mund-Kiefer-Gesichtschirurgen sowie alle in der zahnärztlichen Forschung engagierten Wissenschaftler beteiligen. Internationale Teilnehmer müs- sen über eine gleichwertige akademische Ausbildung verfügen. Zulässige Formate sind veröffentlichte oder angenommene Originalarbeiten in einem international angesehenen Journal mit Impact-Faktor sowie Habilitationsschriften auf den Gebieten der zahnärztlichen Implantologie und Implantatprothetik. Eine Veröffentlichung darf nicht länger als 2 Jahre zurückliegen. Gleiches gilt für das Datum der Habilitation bei Einreichung einer Habilitationsschrift. Die Arbeiten müssen in 5 Exemplaren in deutscher oder englischer Sprache druckfertig geliefert werden an die DGZI Geschäftsstelle, Implant Dentistry Award 2016, Paulusstraße 40237, Düsseldorf. Letzter Abgabetermin ist der 30.06.2016.

Nach einer Pressemitteilung der Deutsche Gesellschaft für Zahnärztliche Implantologie e.V., Düsseldorf 